Avec la révision du règlement de la Commission de déontologie de la $\mathrm{FMH}$, la $\mathrm{FMH}$, conjointement avec les organisations de base, entend uniformiser les règles fondamentales et accélérer les procédures. Un groupe de travail, d'ailleurs composé de représentants tant des organisations de base que de la $\mathrm{FMH}$, a révisé le règlement qui a été adopté par le Comité central. La Chambre médicale a de plus adapté le Code de déontologie en conséquence. La professionnalisation des organes de la FMH est l'objectif stratégique à l'origine de cette révision.

Dr med. Jürg Schlup, président de la FMH

\title{
Nouveau règlement pour la Com- mission de déontologie de la FMH
}

\author{
Anne-Sylvie Thiébaud ${ }^{a}$, Michel Meier $^{b}$
}

${ }^{a}$ avocate, division Service juridique de la $\mathrm{FMH}_{;}{ }^{\mathrm{b}}$ avocat, Société des médecins du canton de Soleure

Le règlement de la Commission de déontologie de la FMH a été révisé dans le cadre de la professionnalisation des organes de la FMH. Avec pour objectifs principaux une meilleure uniformisation des règles générales de procédure ainsi que la simplification et l'amélioration de la célérité de la procédure, la révision a également servi à la création d'un modèle de règlement de procédure à disposition des organisations de base.

\section{Introduction}

Le Code de déontologie de la FMH (CoD) et le règlement de la Commission de la déontologie de la FMH (CdD FMH) sont entrés en vigueur en 1997. Au quotidien, ces dispositions sont appliquées par les commissions de déontologie des organisations de base (sociétés cantonales de médecine, ASMAC et AMDHS, $1^{\text {res }}$ instances) ainsi que la CdD FMH ( $2^{\mathrm{e}}$ instance), lesquelles interprètent leur contenu afin que le Code de déontologie, qui traite non seulement des relations du médecin avec son patient et ses confrères mais également du comportement du praticien vis-à-vis de la société et de ses partenaires de la santé publique, soit respecté.

En 2013, la division Service juridique de la FMH a rencontré les commissions de déontologie cantonales pour faire un bilan intermédiaire. Le besoin de clarifier les règles de procédure pour l'application du $\mathrm{CoD}$ a été établi, tout comme la nécessité de créer une banque de données des décisions anonymisées des commissions de déontologie. La création d'un modèle de règlement a aussi été plébiscitée.

L'aide à la professionnalisation des commissions de déontologie dans les organisations de base étant une des mesures prévues dans le cadre de la stratégie de la FMH 2013-2016, le Comité central de la FMH a autorisé la formation d'un groupe de travail en janvier 2014 pour proposer des modifications dans le règlement de la $\mathrm{CdD} F M H$, créer un modèle de règlement de procédure à destination des organisations de base et mettre en place une banque de données des décisions anony- misées des commissions de déontologie $\left(1^{\text {re }}\right.$ et $2^{\mathrm{e}}$ instances). Sous la direction du Docteur Jürg Schlup, le groupe de travail "Code de déontologie» se composait de la Doctoresse Marianne Winterhalter (ancienne présidente de la Commission de déontologie de Bâle-Ville), du Docteur René Raggenbass (président de la Commission de déontologie du canton du Valais), du Docteur Urs Strebel (ancien vice-président de la Commission de déontologie de la FMH), de Monsieur Michel Meier (avocat de la Société de médecine du canton de Soleure et secrétaire juridique de la Commission de déontologie cantonale) ainsi que de Madame Anne-Sylvie Thiébaud (avocate à la division Service juridique de la FMH et responsable du domaine de la déontologie).

\section{Démarches et objectifs de la révision}

Le groupe de travail mandaté a préparé un nouveau règlement de procédure pour la Commission de déontologie de la FMH ainsi qu'un modèle de règlement de procédure à disposition des organisations de base qui le souhaitent. Les organisations de base ainsi que les commissions de déontologie ont été consultées à deux reprises, soit à l'automne 2014 et au début de l'année 2015. Suite aux consultations des organisations de base et compte tenu de la pratique, les principaux objectifs, soit d'une part une meilleure uniformisation des règles de base et d'autre part la simplification et l'amélioration de la célérité de la procédure, ont pu être mis en œuvre. Bon nombre de ces dispositions, à savoir les 
articles 4 à 27 du nouveau règlement de la CdD FMH, seront applicables non seulement devant la CdD FMH mais également auprès des commissions de déontologie cantonales. En effet, aux termes de l'art. 43 al. $6 \mathrm{du}$ CoD, «les dispositions générales de procédure du règlement de la Commission de déontologie de la FMH sont également valables pour la procédure devant la commission de déontologie des sociétés cantonales de médecine, de l'ASMAC et de l'AMDHS".

Dans la poursuite de ces objectifs, les principaux changements dans le règlement de la $\mathrm{CdD}$ FMH peuvent se résumer ainsi:

- la compétence de la commission de déontologie en cas de changement d'organisation de base ou de perte de la qualité de membre n'est pas affectée (art. 5)

- afin que la procédure de conciliation soit plus favorisée, une procédure en deux étapes est en règle générale prévue en première instance (art. 6)

- précision des règles pour le dénonciateur et le demandeur (art. 7) ainsi que des règles applicables pour le patient partie à la procédure (art. 8)

- interruption de la prescription (art. 10)

- clarification s'agissant de la langue de la procédure (art. 11)

- abolition des féries judiciaires et un seul échange d'écriture prévu (art. 21), possibilité de ne communiquer que le dispositif de la décision (art. 25)

- uniformisation et création d'un principe s'agissant des frais de procédure (art. 23)

- création et insertion des décisions dans une banque de données (art. 27)

S'agissant des règles applicables uniquement devant l'autorité de recours (CdD FMH), on peut encore relever deux changements principaux:

- modification et clarification de la teneur et la forme du recours (art. 32)

- abolition de la séance de conciliation tout en prévoyant la possibilité de tenir des débats oraux si nécessaire (art. 34)

En juin 2015, le Comité central de la FMH a approuvé la révision du règlement de la $\mathrm{CdD} F M H$ et la mise à disposition du modèle de règlement de procédure aux organisations de base. Le nouveau règlement est entré en vigueur le 29 août 2015; les documents sont accessibles sur le site Internet de la FMH [1].

Correspondance:

FMH / Division Service

juridique

Elfenstrasse 18

CH-3000 Berne 15

Tél. 0313591111

Fax 0313591112

lex[at]fmh.ch

\section{Modification de l'art. 44 du Code de déontologie de la FMH}

En parallèle et dans le cadre de cette révision, l'art. 44 du Code de déontologie de la FMH a été modifié par la
Chambre Médicale du 7 mai 2015 [2]. En cas de lacune dans le règlement de la CdD FMH ou des organisations de base, ce sont désormais les règles du Code de procédure civile suisse (CPC) qui s'appliquent en lieu et place de la loi fédérale sur la procédure administrative. La procédure civile est celle qui se rapproche le plus de la procédure appliquée devant les commissions de déontologie. Ces dernières ont un statut particulier puisqu'elles ne sont ni des autorités pénales, ni des autorités civiles ni même administratives. Elles prononcent toutefois des sanctions disciplinaires. Si l'art. $44 \mathrm{CoD}$ renvoyait aux règles de la procédure administrative, ce renvoi était lié à des raisons historiques, faute d'uniformisation de la procédure civile en Suisse. Le CPC est entré en vigueur en 2011 pour toute la Suisse et sera désormais appliqué aux procédures devant les commissions de déontologie lorsqu'une question n'aura pas été réglée dans le CoD ou dans le règlement de procédure. Sans contestation du procès-verbal de la Chambre Médicale, l'art. $44 \mathrm{CoD}$ entre en vigueur le 29 août 2015.

\section{Conclusion}

Sans être exhaustifs ni parachevés, les nouveaux outils à disposition des commissions de déontologie cantonales et de la FMH devraient contribuer à la professionnalisation de ces organes et à une application plus uniforme du CoD. La future banque de données des décisions anonymisées des commissions de déontologie, uniquement à disposition de ces dernières, devrait être opérationnelle au printemps 2016 et venir ainsi compléter les outils susmentionnés. Les interactions du groupe de travail interdisciplinaire, composé tant de médecins que de juristes de la première et de la deuxième instance, tout comme la consultation des organisations de base ont permis de soulever plusieurs lacunes et problèmes rencontrés dans l'application du CoD. Elles ont également rendu possible la mise en œuvre de solutions qui se veulent - dans la mesure du possible et dans le respect des principes juridiques de base-pragmatiques.

\section{Références}

1 www.fmh.ch/fr/de_la_fmh/bases_juridiques/code_deontologie. html

2 Henzen M. Procès-verbal de la première Chambre médicale de 2015. Bull Méd Suisses. 2015;96(27-28):992-1015 